\title{
Remark on weighted approximation properties of generalized Picard operators
}

\author{
B. Yilmaz, G. Başcanbaz Tunca, and A. Aral
}




\title{
REMARK ON WEIGHTED APPROXIMATION PROPERTIES OF GENERALIZED PICARD OPERATORS
}

\author{
B. YILMAZ, G. BAŞCANBAZ-TUNCA, AND A. ARAL
}

Received 18 September, 2012

\begin{abstract}
In this article, we still hold the study of the generalized Picard operators $P_{\lambda, \beta}(f ; q)$ depending on nonisotropic $\beta$-distance given in [3]. By continuing to deal with the nonisotropic weighted $L_{p, \beta}\left(\mathbb{R}^{n}\right)$ space defined in [17], we introduce a new weighted $L_{p, \beta}$ modulus of continuity depending on the nonisotropic distance to obtain the weighted rate of convergence. We show that weighted convergence rate of $P_{\lambda, \beta}(f ; q)$ to $f$ can be made better not only depending on the chosen $q$ but also the choice of $\beta$. We get that $P_{\lambda, \beta}(f ; q)$ satisfy the global smoothness preservation property via weighted $L_{p, \beta}$ modulus of continuity. Also we give direct approximation property of the generalized Picard operators $P_{\lambda, \beta}(f ; q)$ with respect to nonisotropic weighted norm.
\end{abstract}

2010 Mathematics Subject Classification: 42B20; 45P05

Keywords: generalized Picard singular integral nonisotropic distance, Lebesgue point

\section{INTRODUCTION}

The $q$-analysis concept is comprehensive in appoximation theory, especially in the study of various sequences of linear positive operators and singular integral operators, such as Picard and Gauss-Weierstrass singular integral operators (see $[8,10$, 11]). We previously studied Picard singular operators in multivariate setting defined as in [17]. For a general framework related to the classical Picard operators $[2,4,5,13]$ and [14] may be referred. In [7] and [6], multivarite Picard operators and Gauss-Weierstrass operators with kernels including nonisotropic distance were introduced and pointwise convergence result were given. Latterly, in [8], Aral introduced a generalization of the Picard singular singular integral operators (see [4]) by using the $q$-analogue of the Euler Gamma integral and entitled that operators as $q$ - Picard singular integral operators. Also, the author showed that these generalized operators have a more flexible rate of convergence than the classical Picard singular integral operators. In [3], Anasstasiou and Aral introduced the multivariate variant of the $q$-Picard singular integral operators $P_{\lambda, \beta}(f)$ depending on nonisotropic norm (see Definition 3). They introduced a suitable modulus of continuity depending on nonisotropic distance with supremum norm to measure the rate of convergence. Also, 
they proved the global smoothness preservation property of these operators. Also generalized potential-type integral operators with nonisotropic kernel was studied in [9].

In [17] we defined a weighted $L_{p, \beta}\left(\mathbb{R}^{n}\right)$ space (see Definition 2) and the concept of nonisotropic $\beta$-Lebesgue point. Then we obtained a pointwise convergence result for the family of $P_{\lambda, \beta}(f ; q)$ to $f$ for $f \in L_{p, \beta}\left(\mathbb{R}^{n}\right)$. We also studied the rate of this pointwise convergence. Convergence in the norm of this space was also studied.

In this work, we introduce a weighted $L_{p, \beta}$ modulus of continuity (see 2.1) depending on nonisotropic distance in order to measure the rate of convergence in the norm of $L_{p, \beta}$. Also, we show that the operators $P_{\lambda, \beta}(f ; q)$ satisfy the global smoothness preservation property via new weighted $L_{p, \beta}$ modulus of continuity. Futhermore, we show that these operators retain direct approximation result in weighted $L_{p, \beta}\left(\mathbb{R}^{n}\right)$ space with respect to the nonisotropic weighted norm.

Now, we give the concept of the nonisotropic $\beta$-distance. Let $n \in \mathbb{N}$ and $\beta_{1}$, $\beta_{2}, \cdots, \beta_{n}$ be positive numbers with $|\beta|=\beta_{1}+\beta_{2}+\cdots+\beta_{n}$ and

$$
\|\mathbf{x}\|_{\beta}=\left(\left|x_{1}\right|^{\frac{1}{\beta_{1}}}+\cdots+\left|x_{n}\right|^{\frac{1}{\beta_{n}}}\right)^{\frac{|\beta|}{n}}, \mathbf{x} \in \mathbb{R}^{n} .
$$

The expression $\|\mathbf{x}\|_{\beta}$ is called the nonisotropic $\beta$-distance between $\mathbf{x}$ and $\mathbf{0}$. Note that this distance has the following properties of homogeneity for positive $t$ :

$$
\left(\left|t^{\beta_{1}} x_{1}\right|^{\frac{1}{\beta_{1}}}+\cdots+\left|t^{\beta_{n}} x_{n}\right|^{\frac{1}{\beta_{n}}}\right)^{\frac{|\beta|}{n}}=t^{\frac{|\beta|}{n}}\|\mathbf{x}\|_{\beta} .
$$

Also, nonisotropic $\beta$-distance has following properties.

(1) $\|\mathbf{x}\|_{\beta}=0 \Leftrightarrow \mathbf{x}=0$,

(2) $\left\|t^{\beta} \mathbf{x}\right\|_{\beta}=t^{\frac{|\beta|}{n}}\|\mathbf{x}\|_{\beta}$,

(3) $\|\mathbf{x}+\mathbf{y}\|_{\beta} \leq M_{\beta}\left(\|\mathbf{x}\|_{\beta}+\|\mathbf{y}\|_{\beta}\right)$,

$$
\text { where } \beta_{\min }=\min \left\{\beta_{1}, \beta_{2}, \ldots \beta_{n}\right\} \text { and } M_{\beta}=2^{\left(1+\frac{1}{\beta_{\min }}\right) \frac{|\beta|}{n}} \text {, (see [16]) }
$$

It can be seen that nonisotropic $\beta$-distance becomes the ordinary Euclidean distance $|\mathbf{x}|$ for $\beta_{i}=\frac{1}{2}, i=1,2, \ldots, n$. Also, this distance does not satisfy the triangle inequality. Throughout the paper, we need the following notations of $q-$ calculus.

The $q$-extension of exponential function $e^{x}$ is

$$
E_{q}(x):=\sum_{n=0}^{\infty} \frac{q^{\frac{n(n-1)}{2}}}{(q ; q)_{n}} x^{n}=(-x ; q)_{\infty},
$$

with $(a ; q)_{n}=\prod_{k=0}^{n-1}\left(1-a q^{k}\right)$ and $(-x ; q)_{\infty}=\prod_{k=0}^{\infty}\left(1+x q^{k}\right)$. 
For $q>0, q-$ number is

$$
[\lambda]_{q}= \begin{cases}\frac{1-q^{\lambda}}{1-q}, & q \neq 1 \\ \lambda, & q=1\end{cases}
$$

for all nonnegative $\lambda$. If $\lambda$ is an integer, i.e. $\lambda=n$ for some $n$, we write $[n]_{q}$ and call it $q$-integer. Also, we define a $q$-factorial as

$$
[n]_{q} !=\left\{\begin{array}{ll}
{[n]_{q}[n-1]_{q} \cdots[1]_{q},} & n=1,2, \ldots \\
1 & n=0 .
\end{array} .\right.
$$

For integers $0 \leq k \leq n$, the $q$-binomial coefficients are given by

$$
\left[\begin{array}{l}
n \\
k
\end{array}\right]_{q}=\frac{[n]_{q} !}{[k]_{q} ![n-k]_{q} !}
$$

For details see [15].

Another needed formula is $q$-extension of Euler integral representation for the gamma function given in [12] and [1] for $0<q<1$

$$
c_{q}(x) \Gamma_{q}(x)=\frac{1-q}{\ln q^{-1}} q^{\frac{x(x-1)}{2}} \int_{0}^{\infty} \frac{t^{x-1}}{E_{q}((1-q) t)} d t, \quad \operatorname{Re} x>0
$$

where $\Gamma_{q}(x)$ is the $q$-gamma function defined by

$$
\Gamma_{q}(x)=\frac{(q ; q)_{\infty}}{\left(q^{x} ; q\right)_{\infty}}(1-q)^{1-x}, 0<q<1
$$

and $c_{q}(x)$ satisfies the following conditions:

(1) $c_{q}(x+1)=c_{q}(x)$,

(2) $c_{q}(n)=1, n=0,1,2, \ldots$,

(3) $\lim _{q \rightarrow 1^{-}} c_{q}(x)=1$.

When $x=n+1$ with $n$ is a nonnegative integer, we obtain

$$
\Gamma_{q}(n+1)=[n]_{q} !
$$

In [3], Anasstasiou and Aral introduced the $q$-Picard singular integral operators depending on nonisotropic distance taking the following lemma and (1.2) into consideration.

Lemma 1. For all $\lambda>0, n \in \mathbb{N}$ and $\beta_{i} \in(0, \infty)(i=1,2, \ldots n)$ with $|\beta|=\beta_{1}+\beta_{2}+\cdots+\beta_{n}$ we have

$$
\frac{c(n, \beta, q)}{[\lambda]_{q}^{|\beta|}} \int_{\mathbb{R}^{n}} \mathcal{P}_{\lambda}(\beta, \mathbf{t}) d \mathbf{t}=1,
$$


where

and

$$
\mathcal{P}_{\lambda}(\beta, \mathbf{t})=1 / E_{q}\left(\frac{(1-q)|| \mathbf{t} \|_{\beta}}{[\lambda]_{q}^{\frac{|\beta|}{n}}}\right)
$$

$$
c(n, \beta, q)^{-1}=\frac{n}{2|\beta|} \omega_{\beta, n-1} \Gamma_{q}(n) \frac{\ln q^{-1}}{(1-q) q^{\frac{n(n-1)}{2}}} .
$$

Definition 1. Let $f: \mathbb{R}^{n} \rightarrow \mathbb{R}$ be a function. For $0<q<1, \lambda>0, n \in \mathbb{N}$ and $\beta_{i} \in(0, \infty)(i=1,2, \ldots, n)$ with $|\beta|=\beta_{1}+\beta_{2}+\cdots+\beta_{n}$, the $q$-Picard singular integral of $f$ depending on $\beta$-distance of $f$ is

$$
\begin{aligned}
P_{\lambda, \beta}(f ; q, \mathbf{x}) & \equiv P_{\lambda, \beta}(f ; \mathbf{x}) \\
& :=\frac{c(n, \beta, q)}{[\lambda]_{q}^{|\beta|}} \int_{\mathbb{R}^{n}} f(\mathbf{x}+\mathbf{t}) \mathcal{P}_{\lambda}(\beta, \mathbf{t}) d \mathbf{t},
\end{aligned}
$$

where $\mathcal{P}_{\lambda}(\beta, \mathbf{t})$ and $c(n, \beta, q)$ are defined as in (1.4) and (1.5), respectively.

Note that, if we take $\beta_{i}=\frac{1}{2}, i=1,2, \ldots, n$, it reduces to $P_{\lambda, \frac{1}{2}}(f ; q, \mathbf{x})$ operators introduced in [8]. If we take $q \rightarrow 1$, then $P_{\lambda, \frac{1}{2}}(f ; 1, \mathbf{x})$ operators are classical multivariate Picard singular integral (see [3]).

From [17], we have the following definition and subsequent result.

Definition 2. For fixed $1 \leq p<\infty$, we denote by $L_{p, \beta}\left(\mathbb{R}^{n}\right)$ the non-izotropic weighted space of all real valued functions $f$ defined on $\mathbb{R}^{n}$ for which $\frac{f(\mathbf{x})}{1+\|\mathbf{x}\|_{\beta}}$ is a function such that $p$-th power is Lebesgue integrable. The norm in $L_{p, \beta}\left(\mathbb{R}^{n}\right)$ is defined by the formula

$$
\|f\|_{p, \beta}=\left(\int_{\mathbb{R}^{n}}\left|\frac{f(\mathbf{x})}{1+\|\mathbf{x}\|_{\beta}}\right|^{p} d \mathbf{x}\right)^{\frac{1}{p}} .
$$

Lemma 2. $P_{\lambda, \beta}(f)$ is a linear positive operator from the space $L_{p, \beta}\left(\mathbb{R}^{n}\right)$ into $L_{p, \beta}\left(\mathbb{R}^{n}\right)$. That is

$$
\left\|P_{\lambda, \beta}(f)\right\|_{p, \beta} \leq K(n, \beta, q)\|f\|_{p, \beta},
$$

where

$$
\begin{aligned}
K(n, \beta, q) & =\max \left\{1, M_{\beta}\right\}\left(1+\frac{n}{2|\beta|} c(n, \beta, q) \omega_{\beta, n-1} c_{q}\left(n-\frac{n}{2|\beta|}+2\right)\right. \\
& \left.\times \Gamma_{q}\left(n-\frac{n}{2|\beta|}+2\right) \frac{\ln \frac{1}{q}}{1-q} q^{\frac{\left(n-\frac{n}{2|\beta|}+2\right)\left(n-\frac{n}{2|\beta|}+1\right)}{2}}\right) .
\end{aligned}
$$




\section{Weighted $L_{p, \beta}$ MODUlus of CONTINUity}

We now introduce a weighted $L_{p, \beta}$ modulus of continuity depending on nonisotropic distance in order to measure the rate of convergence by $P_{\lambda, \beta}(f)$ in $L_{p, \beta}$ norm.

Definition 3. Let $f \in L_{p, \beta}\left(\mathbb{R}^{n}\right), n \in \mathbb{N}$ and $\beta_{i} \in(0, \infty), i=1, \ldots, n$, with $|\beta|=$ $\beta_{1}+\beta_{2}+\ldots+\beta_{n}$. For every $\delta>0$, the nonisotropic weighted $L_{p, \beta}$ modulus of continuity of $f$ is defined as

$$
\omega_{p, \beta}(f ; \delta)=\sup _{\|\mathbf{h}\|_{\beta} \leq \delta}\|f(\mathbf{x}+\mathbf{h})-f(\mathbf{x})\|_{p, \beta} .
$$

Analogously, the modulus of continuity defined above has the following property

Lemma 3. Let $f \in L_{p, \beta}\left(\mathbb{R}^{n}\right), n \in \mathbb{N}$ and $\beta_{i} \in(0, \infty), i=1, \ldots, n$, with $|\beta|=$ $\beta_{1}+\beta_{2}+\ldots+\beta_{n}$. For every $\delta>0$ and $c>0$, then

$$
\omega_{p, \beta}\left(f ; c^{\frac{|\beta|}{n}} \delta\right) \leq(1+c) \omega_{p, \beta}(f ; \delta) .
$$

Proof. For $k \in \mathbb{Z}^{+}$, using similar arguments to that of in [3]. Using generalized Minkowsky inequality we have the following

$$
\begin{aligned}
& \omega_{p, \beta}\left(f ; k^{\frac{|\beta|}{n}} \delta\right)=\sup _{\|\mathbf{h}\|_{\beta} \leq \delta}\left\|f\left(\mathbf{x}+k^{\beta} \mathbf{h}\right)-f(\mathbf{x})\right\|_{p, \beta}= \\
& =\sup _{\|\mathbf{h}\|_{\beta} \leq \delta}\left(\iint_{\mathbb{R}^{n}} \mid \frac{\left.\sum_{s=1}^{k}\left[f\left(\mathbf{x}+s^{\beta} \mathbf{h}\right)-f\left(\mathbf{x}+(s-1)^{\beta} \mathbf{h}\right)\right]\right|^{p}}{1+\|\mathbf{x}\|_{\beta}} d \mathbf{x}\right)^{\frac{1}{p}}= \\
& =\sup _{\|\mathbf{h}\|_{\beta} \leq \delta}\left\|\sum_{s=1}^{k}\left[f\left(\mathbf{x}+s^{\beta} \mathbf{h}\right)-f\left(\mathbf{x}+(s-1)^{\beta} \mathbf{h}\right)\right]\right\|_{p, \beta} \\
& \leq \sup _{\|\mathbf{h}\|_{\beta} \leq \delta} \sum_{s=1}^{k}\left\|\left[f\left(\mathbf{x}+s^{\beta} \mathbf{h}\right)-f\left(\mathbf{x}+(s-1)^{\beta} \mathbf{h}\right)\right]\right\|_{p, \beta} \\
& \left.\leq \sum_{s=1\left\|s^{\beta} \mathbf{h}-(s-1)^{\beta} \mathbf{h}\right\|_{\beta} \leq \delta}^{k} \int_{\mathbb{R}^{n}}\left|\underline{\left[f\left(\mathbf{x}+s^{\beta} \mathbf{h}\right)-f\left(\mathbf{x}+(s-1)^{\beta} \mathbf{h}\right)\right]}\right|^{p} d \mathbf{x}\right)^{\frac{1}{p}} \\
& \leq k \omega_{p, \beta}(f ; \delta),
\end{aligned}
$$

where $\left\|s^{\beta} \mathbf{h}-(s-1)^{\beta} \mathbf{h}\right\|_{\beta} \leq\|\mathbf{h}\|_{\beta}$, by $s^{\beta_{i}}-(s-1)^{\beta_{i}} \leq 1$ for $i=1,2, \ldots, n$. Since $\omega_{p, \beta}(f ; \delta)$ is a nondecreasing function of $\delta$, then (2.2) is attained easily. 
Now, we give an estimate by means of nonisotropic weighted $L_{p, \beta}$ modulus of continuity of $f$.

Theorem 1. Let $f: \mathbb{R}^{n} \rightarrow \mathbb{R}, f \in L_{p, \beta}\left(\mathbb{R}^{n}\right)$ and $\omega_{p, \beta}(f ; \delta)<\infty$. Then for any $\delta>0$ we have

$$
\left\|P_{\lambda, \beta}(f)-f\right\|_{p, \beta} \leq \omega_{p, \beta}\left(f ;[\lambda]_{q}^{\frac{|\beta|}{n}}\right) M(n, q, \beta)
$$

where

$$
M(n, q, \beta)=1+\frac{q^{\frac{n(n-1)}{2}} \Gamma_{q}\left(n+\frac{n}{|\beta|}\right) c_{q}\left(n+\frac{n}{|\beta|}\right)}{\Gamma_{q}(n) q^{\frac{\left(n+\frac{n}{|\beta|}\right)\left(n+\frac{n}{|\beta|}-1\right)}{2}}}
$$

and $\omega_{p, \beta}(f ;$.$) is the nonisotropic weighted L_{p, \beta}$ modulus of continuity of $f$ given by $(2.1)$.

Proof. From (1.4) and (1.6), by the same reasoning as in Theorem 1 of [3], we have

$$
\begin{aligned}
\left\|P_{\lambda, \beta}(f ; \mathbf{x})-f(\mathbf{x})\right\|_{p, \beta} & \leq \frac{c(n, \beta, q)}{[\lambda]_{q}^{|\beta|}} \int_{\mathbb{R}^{n}}\left(\int_{\mathbb{R}^{n}}\left|\frac{[f(\mathbf{x}+\mathbf{t})-f(\mathbf{x})]}{1+\|\mathbf{x}\|_{\beta}}\right|^{p} d \mathbf{x}\right)^{\frac{1}{p}} \mathcal{P}_{\lambda}(\beta, \mathbf{t}) d \mathbf{t} \\
& \leq \frac{c(n, \beta, q)}{[\lambda]_{q}^{|\beta|}} \int_{\mathbb{R}^{n}} \omega_{p, \beta}\left(f ;\|\mathbf{t}\|_{\beta}\right) \mathcal{P}_{\lambda}(\beta, \mathbf{t}) d \mathbf{t} .
\end{aligned}
$$

Using (2.2) with $c=\frac{\|\mathbf{t}\|_{\beta}^{\frac{n}{|\beta|}}}{[\lambda]_{q}}$ for $\mathbf{t} \in \mathbb{R}^{n}$, we get that

$\omega_{p, \beta}\left(f ;\|\mathbf{t}\|_{\beta}\right)=\omega_{p, \beta}\left(f ;\left(\frac{\|\mathbf{t}\|_{\beta}^{\frac{n}{|\beta|}}}{[\lambda]_{q}}\right)^{\frac{|\beta|}{n}}[\lambda]_{q}^{\frac{|\beta|}{n}}\right) \leq\left(1+\frac{\|\mathbf{t}\|_{\beta}^{\frac{n}{|\beta|}}}{[\lambda]_{q}}\right) \omega_{p, \beta}\left(f ;[\lambda]_{q}^{\frac{|\beta|}{n}}\right)$.

Therefore (2.3) results in

$$
\begin{aligned}
& \left\|P_{\lambda, \beta}(f ; \mathbf{x})-f(\mathbf{x})\right\|_{p, \beta} \leq \\
& \leq \omega_{p, \beta}\left(f ;[\lambda]_{q}^{\frac{|\beta|}{n}}\right) \times\left\{1+\frac{c(n, \beta, q)}{[\lambda]_{q}^{|\beta|+1}} \int_{\mathbb{R}^{n}}\|\mathbf{t}\|_{\beta}^{\frac{n}{|\beta|}} \mathcal{P}_{\lambda}(\beta, \mathbf{t}) d \mathbf{t}\right\} .
\end{aligned}
$$

Applying change of variable $\mathbf{t}=[\lambda]_{q}^{\beta} \mathbf{y}, d \mathbf{t}=[\lambda]_{q}^{|\beta|} d \mathbf{y}$, such that $[\lambda]_{q}^{\beta} \mathbf{y}=\left([\lambda]_{q}^{\beta} y_{1}, \ldots,[\lambda]_{q}^{\beta} y_{n}\right)$ and using the generalized $\beta$-spherical coordinates and 
finally taking (1.2) and (1.5) into consideration to the result, last inequality reduces to

$$
\begin{aligned}
& \left\|P_{\lambda, \beta}(f ; \mathbf{x})-f(\mathbf{x})\right\|_{p, \beta} \leq \\
& \leq \omega_{p, \beta}\left(f ;[\lambda]_{q}^{\frac{|\beta|}{n}}\right) \times\left\{1+c(n, \beta, q) \int_{\mathbb{R}^{n}} \frac{\|\mathbf{y}\|_{\beta}^{\frac{n}{|\beta|}}}{E_{q}\left((1-q)\|\mathbf{y}\|_{\beta}\right)} d \mathbf{y}\right\} \\
& =\omega_{p, \beta}\left(f ;[\lambda]_{q}^{\mid \frac{|\beta|}{n}}\right) \times\left\{1+c(n, \beta, q) \int_{0}^{\infty} \int_{S^{n-1}} \frac{u^{2|\beta|-1} u^{2} \Omega_{\beta}(\theta) d \theta d u}{E_{q}\left((1-q) u^{\frac{2|\beta|}{n}}\right)}\right\} \\
& =\omega_{p, \beta}\left(f ;[\lambda]_{q}^{\frac{|\beta|}{n}}\right) \times\left\{1+\frac{c(n, \beta, q)}{[\lambda]_{q}[\lambda]_{q}^{|\beta|}} \omega_{\beta, n-1} \int_{0}^{\infty} \frac{u^{n+\frac{n}{|\beta|}-1} d u}{E_{q}((1-q) u)}\right\} \\
& =\omega_{p, \beta}\left(f ;[\lambda]_{q}^{\frac{|\beta|}{n}}\right) M(n, q, \beta),
\end{aligned}
$$

where

$$
M(n, q, \beta)=1+\frac{q^{\frac{n(n-1)}{2}} \Gamma_{q}\left(n+\frac{n}{|\beta|}\right) c_{q}\left(n+\frac{n}{|\beta|}\right)}{\Gamma_{q}(n) q^{\frac{\left(n+\frac{n}{|\beta|}\right)\left(n+\frac{n}{|\beta|}-1\right)}{2}}} .
$$

Now, using weighted $L_{p}$-modulus of continuity defined by (2.1), we show that the $q$-Picard integral operators depending on the nonisotropic $\beta$-distance given by (1.6) satisfy the global smoothness preservation property.

Theorem 2. Let $f: \mathbb{R}^{n} \rightarrow \mathbb{R}$, and $\omega_{p, \beta}(f ; \delta)<\infty$ for any $\delta>0$ and $\beta_{i} \in$ $(0, \infty),(i=1, \ldots, n)$ with $|\beta|=\beta_{1}+\beta_{2}+\ldots+\beta_{n}$, such that $P_{\lambda, \beta}(f ; \mathbf{x}) \in \mathbb{R}$ for $0<q<1$. Then we have

$$
\omega_{p, \beta}\left(P_{\lambda, \beta}(f ; .) ; \delta\right) \leq L(n, q, \lambda, \beta) \omega_{p, \beta}(f ; \delta),
$$

where

$L(n, q, \lambda, \beta)=\max \left\{1, M_{\beta}\right\} \times\left(1+\frac{n}{2|\beta|} c(n, \beta, q)[\lambda]_{q}^{\frac{|\beta|}{n}} \omega_{\beta, n-1} \Gamma_{q}(n+1) \frac{\ln q^{-1}}{1-q} q^{-\frac{n(n+1)}{2}}\right)$

in which $M_{\beta}$ is given by property (3) of nonisotropic distance.

Proof. From (1.6) we can write

$\mathcal{P}_{\lambda, \beta}(f ; \mathbf{x}+\mathbf{h})-\mathcal{P}_{\lambda, \beta}(f ; \mathbf{x})=\frac{c(n, \beta, q)}{[\lambda]_{q}^{|\beta|}} \int_{\mathbb{R}^{n}}(f(\mathbf{x}+\mathbf{t}+\mathbf{h})-f(\mathbf{x}+\mathbf{t})) \mathcal{P}_{\lambda}(\beta, \mathbf{t}) d \mathbf{t}$. 
Taking $L_{p, \beta}\left(\mathbb{R}^{n}\right)$ norm and using generalized Minkowsky inequality, we obtain that

$$
\begin{aligned}
& \left(\int_{\mathbb{R}^{n}}\left|\frac{\mathcal{P}_{\lambda, \beta}(f ; \mathbf{x}+\mathbf{h})-\mathcal{P}_{\lambda, \beta}(f ; \mathbf{x})}{1+\|\mathbf{x}\|_{\beta}}\right|^{p} d \mathbf{x}\right)^{\frac{1}{p}}= \\
& =\left(\int_{\mathbb{R}^{n}}\left|\frac{c(n, \beta, q)}{[\lambda]_{q}^{|\beta|}} \int_{\mathbb{R}^{n}} \frac{(f(\mathbf{x}+\mathbf{t}+\mathbf{h})-f(\mathbf{x}+\mathbf{t})) \mathcal{P}_{\lambda}(\beta, \mathbf{t}) d \mathbf{t}}{1+\|\mathbf{x}\|_{\beta}}\right|^{p} d \mathbf{x}\right)^{\frac{1}{p}} \\
& =\frac{c(n, \beta, q)}{[\lambda]_{q}^{|\beta|}}\left(\int_{\mathbb{R}^{n}}\left|\frac{1}{1+\|\mathbf{x}\|_{\beta}} \int_{\mathbb{R}^{n}}(f(\mathbf{x}+\mathbf{t}+\mathbf{h})-f(\mathbf{x}+\mathbf{t})) \mathcal{P}_{\lambda}(\beta, \mathbf{t}) d \mathbf{t}\right|^{p} d \mathbf{x}\right)^{\frac{1}{p}} \\
& \leq \frac{c(n, \beta, q)}{[\lambda]_{q}^{|\beta|}} \int_{\mathbb{R}^{n}}\left(\int_{\mathbb{R}^{n}}\left|\frac{(f(\mathbf{x}+\mathbf{t}+\mathbf{h})-f(\mathbf{x}+\mathbf{t}))}{1+\|\mathbf{x}\|_{\beta}}\right|^{p} d \mathbf{x}\right)^{\frac{1}{p}} \mathcal{P}_{\lambda}(\beta, \mathbf{t}) d \mathbf{t} . \\
& =\frac{c(n, \beta, q)}{[\lambda]_{q}^{|\beta|}} \int_{\mathbb{R}^{n}}\left(\int_{\mathbb{R}^{n}}\left|\frac{f(\mathbf{x}+\mathbf{h})-f(\mathbf{x})}{1+\|\mathbf{x}-\mathbf{t}\|_{\beta}}\right|^{p} d x\right)^{\frac{1}{p}} \mathcal{P}_{\lambda}(\beta, \mathbf{t}) d \mathbf{t}
\end{aligned}
$$

From the property (3), we have

$$
\begin{aligned}
\frac{1}{1+\|\mathbf{x}\|_{\beta}} \frac{1+\|\mathbf{x}\|_{\beta}}{1+\|\mathbf{x}-\mathbf{t}\|_{\beta}} & =\frac{1}{1+\|\mathbf{x}\|_{\beta}} \frac{1+\|\mathbf{x}-\mathbf{t}+\mathbf{t}\|_{\beta}}{1+\|\mathbf{x}-\mathbf{t}\| \|_{\beta}} \\
& \leq \frac{1}{1+\|\mathbf{x}\|_{\beta}} \frac{1+M_{\beta}\left(\|\mathbf{x}-\mathbf{t}\|_{\beta}+\|\mathbf{t}\|_{\beta}\right)}{1+\|\mathbf{x}-\mathbf{t}\|_{\beta}} \\
& =\frac{\max \left\{1, M_{\beta}\right\}}{1+\|\mathbf{x}\|_{\beta}} \frac{\left(1+\|\mathbf{x}-\mathbf{t}\|_{\beta}\right)\left(1+\|\mathbf{t}\|_{\beta}\right)}{1+\|\mathbf{x}-\mathbf{t}\|_{\beta}}
\end{aligned}
$$

Using the above inequality we have

$$
\begin{aligned}
& \left(\int_{\mathbb{R}^{n}}\left|\frac{\mathcal{P}_{\lambda, \beta}(f ; \mathbf{x}+\mathbf{h})-\mathcal{P}_{\lambda, \beta}(f ; \mathbf{x})}{1+\|\mathbf{x}\|_{\beta}}\right|^{p} d \mathbf{x}\right)^{\frac{1}{p}} \leq \\
& \leq \max \left\{1, M_{\beta}\right\} \frac{c(n, \beta, q)}{[\lambda]_{q}^{|\beta|}} \int_{\mathbb{R}^{n}}\left(\int_{\mathbb{R}^{n}}\left|\frac{(f(\mathbf{x}+\mathbf{h})-f(\mathbf{x}))}{1+\|\mathbf{x}\|_{\beta}}\right|^{p} d \mathbf{x}\right)^{\frac{1}{p}}\left(1+\|\mathbf{t}\|_{\beta}\right) \mathcal{P}_{\lambda}(\beta, \mathbf{t}) d \mathbf{t}
\end{aligned}
$$




$$
\begin{aligned}
& \leq \max \left\{1, M_{\beta}\right\}\|f(\mathbf{x}+\mathbf{h})-f(\mathbf{x})\|_{p, \beta} \frac{c(n, \beta, q)}{[\lambda]_{q}^{|\beta|}} \int_{\mathbb{R}^{n}}\left(1+\|\mathbf{t}\|_{\beta}\right) \mathcal{P}_{\lambda}(\beta, \mathbf{t}) d \mathbf{t} \\
& =\max \left\{1, M_{\beta}\right\}\|f(\mathbf{x}+\mathbf{h})-f(\mathbf{x})\|_{p, \beta}\left(1+\frac{c(n, \beta, q)}{[\lambda]_{q}^{|\beta|}} \int_{\mathbb{R}^{n}}\|\mathbf{t}\|_{\beta} \mathcal{P}_{\lambda}(\beta, \mathbf{t}) d \mathbf{t}\right) .
\end{aligned}
$$

Using Lemma 1 and making change of variable $\mathbf{t}=[\lambda]_{q}^{\beta} \mathbf{x}$ we have

$$
\begin{aligned}
& \left(\int_{R^{n}}\left|\frac{\mathcal{P}_{\lambda, \beta}(f ; \mathbf{x}+\mathbf{h})-\mathcal{P}_{\lambda, \beta}(f ; \mathbf{x})}{1+\|\mathbf{x}\|_{\beta}}\right|^{p} d \mathbf{x}\right)^{\frac{1}{p}} \leq \\
& \leq \max \left\{1, M_{\beta}\right\}\|f(\mathbf{x}+\mathbf{h})-f(\mathbf{x})\|_{p, \beta} \times\left(1+c(n, \beta, q)[\lambda]_{q}^{\frac{|\beta|}{n}} \int_{\mathbb{R}^{n}} \frac{\|\mathbf{x}\|_{\beta}}{E_{q}\left((1-q)\|\mathbf{x}\|_{\beta}\right)} d \mathbf{x}\right) .
\end{aligned}
$$

We use generalized $\beta$-spherical coordinates ([16]) and consider the transformation

$$
\begin{aligned}
x_{1} & =\left(u \cos \theta_{1}\right)^{2 \beta_{1}} \\
x_{2} & =\left(u \sin \theta_{1} \cos \theta_{2}\right)^{2 \beta_{2}} \\
\vdots & \\
x_{n-1} & =\left(u \sin \theta_{1} \sin \theta_{2} \cdots \sin \theta_{n-2} \cos \theta_{n-1}\right)^{2 \beta_{n-1}} \\
x_{n} & =\left(u \sin \theta_{1} \sin \theta_{2} \cdots \sin \theta_{n-1}\right)^{2 \beta_{n},},
\end{aligned}
$$

where $0 \leq \theta_{1}, \theta_{2}, \cdots, \theta_{n-2} \leq \pi, 0 \leq \theta_{n-1} \leq 2 \pi, u \geq 0$. Denoting the Jacobian of this transformation by $J_{\beta}\left(u, \theta_{1}, \ldots, \theta_{n-1}\right)$ we obtain

$$
J_{\beta}\left(u, \theta_{1}, \ldots, \theta_{n-1}\right)=u^{2|\beta|-1} \Omega_{\beta}(\theta),
$$

where $\Omega_{\beta}(\theta)=2^{n} \beta_{1} \ldots \beta_{n} \prod_{j=1}^{n-1}\left(\cos \theta_{j}\right)^{2 \beta_{j}-1}\left(\sin \theta_{j}\right)^{\sum_{k=j}^{j+1} 2 \beta_{k}-1}$. We can easily see that the integral

$$
\omega_{\beta, n-1}=\int_{S^{n-1}} \Omega_{\beta}(\theta) d \theta
$$

is finite, where $S^{n-1}$ is the unit sphere in $\mathbb{R}^{n}$.

Thus we have

$$
\left(\int_{R^{n}}\left|\frac{\mathcal{P}_{\lambda, \beta}(f ; \mathbf{x}+\mathbf{h})-\mathcal{P}_{\lambda, \beta}(f ; \mathbf{x})}{1+\|\mathbf{x}\|_{\beta}}\right|^{p} d \mathbf{x}\right)^{\frac{1}{p}} \leq
$$




$$
\begin{aligned}
& \leq \max \left\{1, M_{\beta}\right\}\|(f(\mathbf{x}+\mathbf{h})-f(\mathbf{x}))\|_{p, \beta} \\
& \times\left(1+c(n, \beta, q)[\lambda]_{q}^{\frac{|\beta|}{n}} \int_{0}^{\infty} \int_{S^{n-1}} \frac{u^{2|\beta|+\frac{2|\beta|}{n}-1} \Omega_{\beta}(\theta) d \theta d u}{E_{q}\left((1-q) u^{\frac{2|\beta|}{n}}\right)}\right) \\
& \leq \max \left\{1, M_{\beta}\right\}\|(f(\mathbf{x}+\mathbf{h})-f(\mathbf{x}))\|_{p, \beta} \\
& \times\left(1+\frac{n}{2|\beta|} c(n, \beta, q)[\lambda]_{q}^{\frac{|\beta|}{n}} \omega_{\beta, n-1} \int_{0}^{\infty} \frac{u^{n} d u}{E_{q}((1-q) u)}\right) \\
& \leq \max \left\{1, M_{\beta}\right\}\|(f(\mathbf{x}+\mathbf{h})-f(\mathbf{x}))\|_{p, \beta} \\
& \times\left(1+\frac{n}{2|\beta|} c(n, \beta, q)[\lambda]_{q}^{\mid \frac{|\beta|}{n}} \omega_{\beta, n-1} \Gamma_{q}(n+1) \frac{\ln q^{-1}}{1-q} q^{-\frac{n(n+1)}{2}}\right)
\end{aligned}
$$

Finally we find that

$$
\left\|\mathcal{P}_{\lambda, \beta}(f ; \mathbf{x}+\mathbf{h})-\mathcal{P}_{\lambda, \beta}(f ; \mathbf{x})\right\|_{p, \beta} \leq\|(f(\mathbf{x}+\mathbf{h})-f(\mathbf{x}))\|_{p, \beta} L(n, q, \lambda, \beta)
$$

proving (2.4).

Now we give direct approximation property of $P_{\lambda, \beta}(f ; q)$ with respect to nonisotropic weighted norm.

Theorem 3. Let $f \in L_{p, \beta}\left(\mathbb{R}^{n}\right), n \in \mathbb{N}$ and $\beta_{i} \in(0, \infty), i=1, \ldots, n$, with $|\beta|=$ $\beta_{1}+\beta_{2}+\ldots+\beta_{n}$. We have

$$
\lim _{\lambda \rightarrow 0}\left\|\mathcal{P}_{\lambda, \beta}(f ; \mathbf{x})-f(\mathbf{x})\right\|_{p, \beta}=0 .
$$

Proof. Taking Lemma 1 and generalized Minkowsky inequality into consideration, we get

$$
\begin{aligned}
\left\|P_{\lambda, \beta}(f ; \mathbf{x})-f(x)\right\|_{p, \beta} & \leq \frac{c(n, \beta, q)}{[\lambda]_{q}^{|\beta|}} \int_{\mathbb{R}^{n}}\left(\int_{\mathbb{R}^{n}}\left|\frac{[f(\mathbf{x}+\mathbf{t})-f(\mathbf{x})]}{1+\|\mathbf{x}\|_{\beta}}\right|^{p} d \mathbf{x}\right)^{\frac{1}{p}} \mathcal{P}_{\lambda}(\beta, \mathbf{t}) d \mathbf{t} \\
& =\frac{c(n, \beta, q)}{[\lambda]_{q}^{|\beta|}} \int_{\mathbb{R}^{n}}\|f(\mathbf{x}+\mathbf{t})-f(\mathbf{x})\|_{p, \beta} \mathcal{P}_{\lambda}(\beta, \mathbf{t}) d \mathbf{t} .
\end{aligned}
$$

For a $\delta>0$ we can write

$$
\begin{aligned}
\left\|P_{\lambda, \beta}(f ; \mathbf{x})-f(x)\right\|_{p, \beta} & \leq \frac{c(n, \beta, q)}{[\lambda]_{q}^{|\beta|}} \int_{\|\mathbf{t}\|_{\beta} \leq \delta}\|f(\mathbf{x}+\mathbf{t})-f(\mathbf{x})\|_{p, \beta} \mathcal{P}_{\lambda}(\beta, \mathbf{t}) d \mathbf{t} \\
& +\frac{c(n, \beta, q)}{[\lambda]_{q}^{|\beta|}} \int_{\|\mathbf{t}\|_{\beta}>\delta}\|f(\mathbf{x}+\mathbf{t})-f(\mathbf{x})\|_{p, \beta} \mathcal{P}_{\lambda}(\beta, \mathbf{t}) d \mathbf{t}
\end{aligned}
$$




$$
=L_{1}(\lambda)+L_{2}(\lambda) \text {. }
$$

From Lemma 1 and definition of weighted $L_{p, \beta}$ modulus of continuity, we have

$$
\begin{aligned}
L_{1}(\lambda) & \leq \omega_{p, \beta}(f ; \delta) \frac{c(n, \beta, q)}{[\lambda]_{q}^{|\beta|}} \int_{\|\mathbf{t}\|_{\beta} \leq \delta} \mathcal{P}_{\lambda}(\beta, \mathbf{t}) d \mathbf{t} \\
& =\omega_{p, \beta}(f ; \delta) \frac{c(n, \beta, q)}{[\lambda]_{q}^{|\beta|}} \int_{\mathbb{R}^{n}} \mathcal{P}_{\lambda}(\beta, \mathbf{t}) d \mathbf{t}=1,
\end{aligned}
$$

which implies that

$$
\lim _{\lambda \rightarrow 0} L_{1}(\lambda)=0
$$

Since

we can write

$$
\begin{aligned}
\|f(\mathbf{x}+\mathbf{t})-f(\mathbf{x})\|_{p, \beta} & =\int_{\mathbb{R}^{n}}\left|\frac{[f(\mathbf{x}+\mathbf{t})-f(\mathbf{x})]}{1+\|\mathbf{x}\|_{\beta}}\right|^{p} d \mathbf{x} \\
& \leq \int_{\mathbb{R}^{n}}\left|\frac{f(\mathbf{x}+\mathbf{t})}{1+\|\mathbf{x}\|_{\beta}}\right|^{p} d \mathbf{x}+\int_{\mathbb{R}^{n}}\left|\frac{f(\mathbf{x})}{1+\|\mathbf{x}\|_{\beta}}\right|^{p} d \mathbf{x} \\
& \leq \max \left\{1, M_{\beta}\right\}\left(1+\|\mathbf{t}\|_{\beta}\right)\|f\|_{p, \beta}+\|f\|_{p, \beta},
\end{aligned}
$$

$$
\begin{aligned}
L_{2}(\lambda) & \leq\left(1+\max \left\{1, M_{\beta}\right\}\right)\|f\|_{p, \beta} \frac{c(n, \beta, q)}{[\lambda]_{q}^{|\beta|}} \int_{\|\mathbf{t}\|_{\beta}>\delta} \mathcal{P}_{\lambda}(\beta, \mathbf{t}) d \mathbf{t} \\
& +\|f\|_{p, \beta} \frac{c(n, \beta, q)}{[\lambda]_{q}^{|\beta|}} \int_{\|\mathbf{t}\|_{\beta}>\delta}\|\mathbf{t}\|_{\beta} \mathcal{P}_{\lambda}(\beta, \mathbf{t}) d \mathbf{t} .
\end{aligned}
$$

Since the first and second term of above inequality tends to zero as $\lambda \rightarrow 0$ (see [17, p.508]), we get

$$
\lim _{\lambda \rightarrow 0} L_{2}(\lambda)=0 .
$$

From (2.5) and (2.6) we have desired result.

Remark 1. Using similar arguments in [3, p.825] we can easily see that $\omega_{p, \beta}(f ; \delta)$ tends to zero as $\delta \rightarrow 0$. As a consequence of Theorem 1, we can say that resulting approximation process turns out to have a weighted rate of convergence which is 
$[\lambda]_{q}^{\frac{|\beta|}{n}}$ at least as good as that classical Picard singular integral operators. Also this weighted convergence rate of $P_{\lambda, \beta}(f ; q)$ to $f$ can be made better not only depending on the chosen $q$ but also the choice of $\beta$. This convergence is shown directly in Theorem 3 .

\section{REFERENCES}

[1] R. Álvarez Nodarse, M. K. Atakishiyeva, and N. M. Atakishiyev, "On $q$-extension of the Hermit Polynomials $H_{n}(x)$ with the continuous orthogonality property on R," Bol. Soc. Mat. Mexicana, vol. 8, no. 3, pp. 127-139, 2002.

[2] G. A. Anastassiou, "Global smoothness preservation by singular integrals," Proyecciones, vol. 14, no. 2 , pp. 83-88, 1995

[3] G. A. Anastassiou and A. Aral, "Generalized Picard singular integrals," Comput. Math. Appl., vol. 57, no. 5, pp. 821-830, 2009.

[4] G. A. Anastassiou and S. G. Gal, Approximation theory. Moduli of continuity and global smoothness preservation. Boston: Birkhäuser, 2000.

[5] G. A. Anastassiou and S. G. Gal, "Convergence of generalized singular integrals to the unit, univariate case," Math. Inequal. Appl., vol. 3, no. 4, pp. 511-518, 2000.

[6] A. Aral, "On convergence of singular integrals with non-isotropic kernels," Commun. Fac. Sci. Univ. Ank., Sér. A1, Math. Stat., vol. 50, no. 1-2, pp. 83-93, 2001.

[7] A. Aral, "On a generalized $\lambda$-Gauss Weierstrass singular integral," Fasc. Math., vol. 35, pp. 23-33, 2005.

[8] A. Aral, "On the generalized Picard and Gauss-Weierstrass singular integrals," J. Comput. Anal. Appl., vol. 8, no. 3, pp. 249-261, 2006.

[9] A. Aral, "Fine limits of generalized potential-type integral operators with nonisotropic kernel," Miskolc Math. Notes, vol. 8, no. 2, pp. 123-134, 2007.

[10] A. Aral, "Pointwise approximation by the generalization of Picard and Gauss-Weierstrass singular integrals," J. Concr. Appl. Math., vol. 6, no. 4, pp. 327-339, 2008.

[11] A. Aral and S. G. Gal, " $q$-generalizations of the Picard and Gauss-Weierstrass singular integrals," Taiwanese J. Math., vol. 12, no. 9, pp. 2501-2515, 2008.

[12] N. M. Atakishiyev and M. K. Atakishiyeva, "A $q$-analogue of the Euler gamma integral," Theor. Math. Phys., vol. 129, no. 1, pp. 1325-1334 (2001); translation from teor. mat. fiz. 129, no. 1, 20-30, 2001.

[13] S. G. Gal, "Degree of approximation of continuous function by some singular integrals," Rev. Anal. Numér. Théor. Approx., vol. XXVII, no. 1, pp. 251-261, 1998.

[14] S. G. Gal, "Remark on degree of approximation of continuous function by some singular integrals," Math. Nachr., vol. 164, pp. 197-199, 1998.

[15] G. Gasper and M. Rahman, Basic hypergeometric series., ser. Encyclopedia of Mathematics and Its Applications. Cambridge: Cambridge University Press, 1990, vol. 34.

[16] V. P. II'in, O. V. Besov, and S. M. Nikolsky, The Integral Representation of Functions and Embedding Theorems. Nauka, 1975.

[17] B. sar Yilmaz, A. Aral, and G. Başcanbaz-Tunca, "Weighted approximation properties of generalized Picard operators," J. Comput. Anal. Appl., vol. 13, no. 3, pp. 499-513, 2011. 


\section{Authors' addresses}

\section{B. Yılmaz}

Ankara University, Faculty of Science, Department of Mathematics, 06100, Tandogan, Ankara, Turkey

E-mail address: basaryilmaz77@yahoo.com

\section{G. Başcanbaz-Tunca}

Ankara University, Faculty of Science, Department of Mathematics, 06100, Tandogan, Ankara, Turkey

E-mail address: tuncaescience.ankara.edu.tr

A. Aral

Kırıkkale University, Faculty of Science and Arts, Department of Mathematics, Yahşihan, Kirikkale, Turkey

E-mail address: aliaral73@yahoo.com 